\title{
Coplanar Repeats by Energy Minimization
}

James Pritts ${ }^{1}$

http://cmp.felk.cvut.cz/ prittjam

Denys Rozumnyi ${ }^{1}$

rozumden@cmp.felk.cvut.cz

\author{
M. Pawan Kumar ${ }^{2}$ \\ http://mpawankumar.info \\ Ondrej Chum ${ }^{1}$ \\ http://cmp.felk.cvut.cz/ chum
}

${ }^{1}$ The Center for Machine Perception

Faculty of Electrical Engineering

Czech Technical University

Prague, CZ

${ }^{2}$ Department of Engineering Science University of Oxford Oxford, UK
This paper proposes an automated method to detect, group and rectify arbitrarily-arranged coplanar repeats via energy minimization. We propose a global energy model for grouping coplanar repeats that combines features that encourage (i) the geometric consistency of repeated coplanar elements, (ii) the appearance similarity of planar repeated elements, (iii) the spatial and color coherence of scene planes, (iv) the spatial and color coherence of the background, (v) and the parsimony of detected coplanar repeat groups and scene planes.
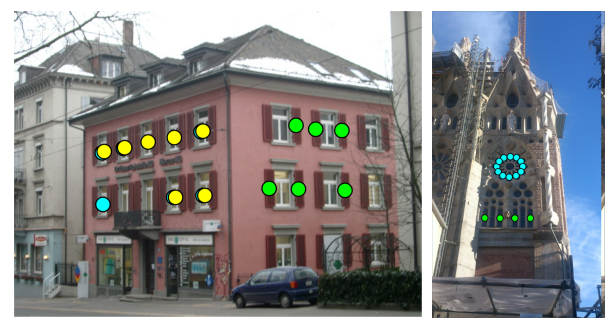

Figure 1: Selected detected coplanar repeats.

A block-coordinate descent framework is proposed for energy minimization that alternately assigns keypoints to coplanar repeats by labeling via a recent variant of $\alpha$-expansion, and regresses the continuous parameters that model the geometries and appearances of coplanar repeat groups and their underlying scene planes.

We introduce a dataset of 113 images containing coplanar repeated patterns with translated, rotated and reflected symmetries that repeat arbitrarily or periodically. The dataset will be made publicly available ${ }^{1}$

To evaluate the performance of the proposed method, we compare against two state-of-theart geometric multi-model fitting methods: JLinkage and MultiRANSAC [3, 4]. The accu- racy of rectifications constructed from vanishing lines computed from estimated coplanar repeat groups are used to compare the methods.

The cumulative distribution of distortions on the dataset (truncated at 10 pixels) is shown in Fig. 2. At 1 pixel of distortion, the proposed method solves $163 \%$ more scene planes than the next best; at 2 pixels, $94 \%$ more; and at 5 pixels, which can be considered a threshold for meaningful rectification, $51 \%$ more scene planes. The proposed energy minimization formulation demonstrates a distinct increase in the quality of rectifications estimated from detected coplanar repeat groups on the evaluated dataset with respect to two state-of-the-art geometric multi-model fitting methods. The advantage can be attributed to the global scene context that is incorporated into the energy functional of the proposed method.

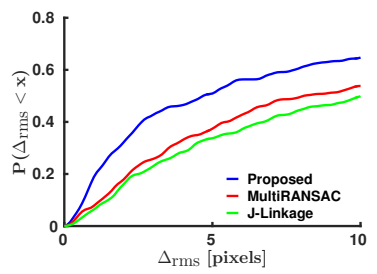

Figure 2: CDF of rectification distortions $\left(\Delta_{\mathrm{rms}}\right)$.

[1] O. Chum and J. Matas. Planar affine rectification from change of scale. In ACCV, 2010.

[2] J. Pritts, O. Chum, and J. Matas. Detection, rectification and segmentation of coplanar repeated patterns. In CVPR, 2014.

[3] R. Toldo and A. Fusiello. Robust multiple structures estimation with j-linkage. In ECCV, 2008.

[4] M. Zuliani, C. Kenney, and Manjunath B. The multiransac algorithm and its application to detect planar homographies. In ICIP, 2005. 